\title{
Effect of a dual trigger on oocyte maturation in young women with decreased ovarian reserve for the purpose of elective oocyte cryopreservation
}

\author{
Se Jeong Kim, Tae Hyung Kim, Jae Kyun Park, Jin Hee Eum, Woo Sik Lee, Sang Woo Lyu \\ Department of Obstetrics and Gynecology, Fertility Center of CHA Gangnam Medical Center, CHA University School of Medicine, Seoul, Korea
}

Objective: The aim of this study was to determine whether co-administration of a gonadotropin-releasing hormone (GnRH) agonist and human chorionic gonadotropin (hCG) for final oocyte maturation improved mature oocyte cryopreservation outcomes in young women with decreased ovarian reserve (DOR) compared with hCG alone.

Methods: Between January 2016 and August 2019, controlled ovarian stimulation (COS) cycles in women (aged $\leq 35$ years, anti-Müllerian hormone $[\mathrm{AMH}]<1.2 \mathrm{ng} / \mathrm{mL}$ ) who underwent elective oocyte cryopreservation for fertility preservation were retrospectively analyzed.

Results: A total of $76 \mathrm{COS}$ cycles were triggered with a GnRH agonist and hCG (the dual group) or hCG alone (the hCG group). The mean age and serum AMH levels were comparable between the two groups. The duration of stimulation, total dose of follicle-stimulating hormone used, and total number of oocytes retrieved were similar. However, the number of mature oocytes retrieved and the oocyte maturation rate were significantly higher in the dual group than in the $\mathrm{hCG}$ group $(p=0.010$ and $p<0.001)$. After controlling for confounders, the dual-trigger method remained a significant factor related to the number of mature oocytes retrieved $(p=0.016)$.

Conclusion: We showed improved mature oocyte collection and maturation rate with the dual triggering of oocyte maturation in young women with DOR. A dual trigger appears to be more beneficial than $\mathrm{hCG}$ alone in terms of mature oocyte cryopreservation for young women with DOR.

Keywords: Dual trigger; GnRH agonist; Fertility preservation; Oocyte; Ovarian reserve

\section{Introduction}

Fertility preservation (FP) techniques consist of oocyte, embryo, and oocyte tissue cryopreservation that can prolong the ability to conceive. The cryopreservation of oocytes matured in vivo that were obtained in controlled ovarian stimulation (COS) cycles has generat-

Received: March 16, 2020 • Revised: July 1, 2020 • Accepted: August 5, 2020 Corresponding author: Sang Woo Lyu

Department of Obstetrics and Gynecology, Fertility Center of CHA Gangnam Medical Center, CHA University School of Medicine, 569, Nonhyeon-ro, Gangnam-gu, Seoul, 06125

Tel: +82-3468-3401 Fax:+82-3468-2609 E-mail: dung5038@cha.ac.kr

This is an Open Access article distributed under the terms of the Creative Commons Attribution Non-Commercial License (http://creativecommons.org/licenses/by-nc/4.0/) which permits unrestricted non-commercial use, distribution, and reproduction in any medium, provided the original work is properly cited. ed many successful results [1,2]. Mature oocyte cryopreservation can now be successfully offered as part of FP programs for patients looking to preserve fertility for medical and social reasons. Thus, this is no longer only an experimental concept [3]. However, the usefulness of cryopreservation of collected immature oocytes before and after in vitro maturation (IVM) is still debatable [4]. Therefore, it is still important for FP to collect a large number of mature oocytes.

Women with decreased ovarian reserve (DOR) are at risk of losing their reproductive ability due to the reduced number and quality of oocytes in the ovary $[5,6]$. Young women with DOR may be candidates for elective oocyte cryopreservation for FP before ovarian failure. However, previous $\mathrm{COS}$ studies were conducted with regards to the in vitro fertilization (IVF) treatment of infertile older women with DOR or young women without DOR (oocyte donors). There is a lack 
of data on obtaining a large number of mature oocytes in young women with DOR.

Meanwhile, one of the most critical steps during COS is the triggering of final oocyte maturation. Human chorionic gonadotropin (hCG) is usually used as a surrogate for the luteinizing hormone surge for final oocyte maturation, resumption of meiosis, and luteinization of the granulosa cells [7]. To reduce the risk of ovarian hyperstimulation syndrome (OHSS), the substitution of hCG with a gonadotropin-releasing hormone $(\mathrm{GnRH})$ agonist was proposed for IVF high responders [8]. However, adverse effects on clinical pregnancy [9] resulted in the emergence of the concept of a dual trigger with a GnRH agonist along with a low dose of hCG [10]. In addition to IVF high responders, the dual-trigger approach has advantages for normal responders in terms of implantation, oocyte collection yield, and oocyte maturation [11]. Furthermore, the dual-trigger approach has been found to improve the oocyte maturation rate in patients with prior low oocyte maturation rates [12]. These benefits have been attributed to the GnRH agonist induced mid-cycle follicle-stimulating hormone (FSH) surge believed to promote oocyte nuclear maturation and cumulus expansion $[13,14]$. Results regarding the number of mature oocytes or oocyte maturation rates following a dual trigger compared with hCG alone in poor responders are inconsistent $[15,16]$. Thus, the aim of this study was to investigate whether a dual trigger with a GnRH agonist along with hCG for final oocyte maturation improved mature oocyte cryopreservation outcomes in young women with DOR compared with hCG alone.

\section{Methods}

This study was conducted retrospectively with approval from the Institutional Review Board of CHA Gangnam Medical Center (IRB No. GCl-19-44). Due to the retrospective design, the requirement for informed consent was waived. This retrospective study included women 35 years old and younger who underwent elective oocyte cryopreservation for FP due to DOR. DOR was defined when the serum anti-Müllerian hormone (AMH) level was lower than $1.2 \mathrm{ng} / \mathrm{mL}$ at the time of $\mathrm{COS}$ initiation [17]. AMH concentrations were measured using an Elecsys AMH immunoassay (Roche Diagnostics, Mannheim, Germany). A total of $122 \mathrm{GnRH}$ antagonist COS cycles performed between January 2016 and August 2019 at a CHA Gangnam Medical Center were selected. The exclusion criteria were women on more than their second cycle, a natural cycle, mild stimulation with oral medication, and retrieval failure. After exclusion, a total of $76 \mathrm{COS}$ cycles were included in the final analysis. The study population was divided into two groups based on the trigger method: a dual trigger with a GnRH agonist and hCG (the dual group, $\mathrm{n}=40$ ) and a trigger of $\mathrm{hCG}$ alone (the $\mathrm{hCG}$ group, $\mathrm{n}=36$ ). Figure 1 depicts the study pop- ulation. We compared the number of mature oocytes and oocyte maturation rates of the two groups and investigated factors associated with the number of mature oocytes retrieved to determine the effect of each triggering method while controlling for confounding factors.

Baseline hormone tests and pelvic ultrasound examinations were performed on day 2 or 3 of the menstrual cycle. COS commenced on the 3rd day of the menstrual cycle using recombinant FSH (rFSH; Gonal-F, Merck-Serono, Darmstadt, Germany; Follitrope, LG Life Sciences, Seoul, Korea) and/or highly purified human menopausal gonadotrophin (hMG; IVF-M HP, LG Life Sciences; Menopur, Ferring, SaintPrex, Switzerland). Patient response was monitored during the COS cycle with serial transvaginal ultrasound examinations, and the gonadotropin dose was adjusted. A GnRH antagonist (Cetrotide, Merck-Serono; Orgalutran, MSD Pharmaceuticals, Courbevoie, France) was added when the leading follicle reached $14 \mathrm{~mm}$ in diameter. When at least one of the follicles reached $18 \mathrm{~mm}$ in diameter, $250 \mu \mathrm{g}$ of recombinant hCG (Ovidrel, Merck-Serono) or coadministration of $0.2 \mathrm{mg}$ of a GnRH agonist (Decapeptyl; Ipsen Pharma, Barcelona, Spain) with $250 \mu \mathrm{g}$ of hCG was administered for final oocyte maturation. The choice of triggering method was made at the physicians' discretion.

Under transvaginal ultrasound guidance, oocyte retrieval was performed 35 to 36 hours after dual or hCG trigger. Oocyte maturity was evaluated using microscopy, and the mature oocyte count was assessed. Mature oocytes had a single polar body with an expanded cumulus-corona. Immature oocytes were cultured for IVM. The oocyte maturation rate was calculated by dividing the number of mature oocytes by the number of total oocytes. After excluding degenerative oocytes, the oocytes were cryopreserved using the vitrifica-

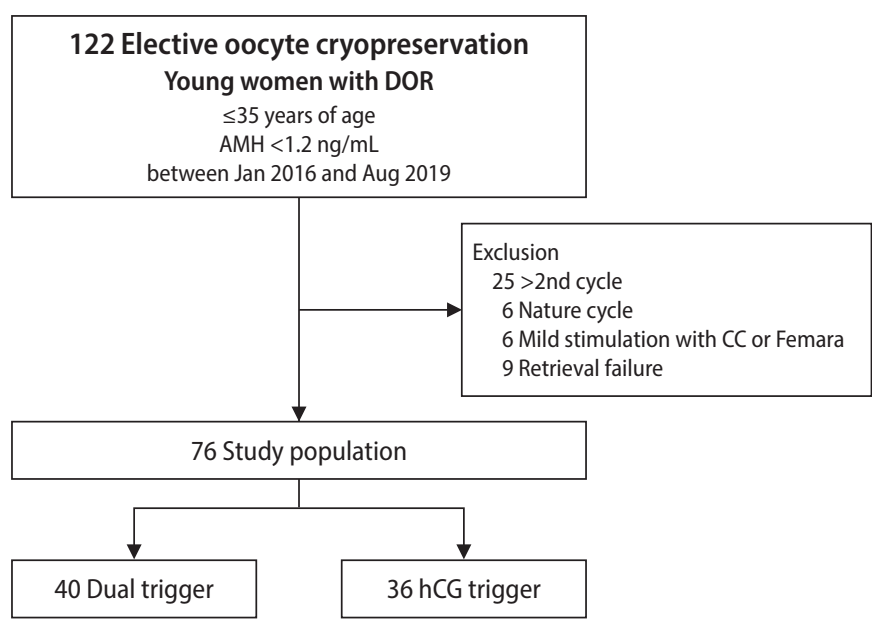

Figure 1. Flow diagram depicting the selection of the study population. DOR, decreased ovarian reserve; $\mathrm{AMH}$, anti-Müllerian hormone; CC, clomiphene citrate; $\mathrm{hCG}$, human chorionic gonadotropin. 
tion method.

All statistical analyses were performed using IBM SPSS ver. 25.0 (IBM Corp., Armonk, NY, USA). Continuous variables are presented as mean \pm standard deviation, and categorical variables are presented as numbers with percentages. Differences between the two groups were analyzed using the Student $t$-test or the Pearson chi-square test. Furthermore, we used univariate analysis to identify factors related to the number of mature oocytes retrieved. Multivariate analysis was performed to examine the adjusted effect of related factors. Subgroup analysis according to the COS regimen was performed using the Mann-Whitney test, and $p$-values $<0.05$ were considered to indicate statistical significance.

\section{Results}

The clinical characteristics of the study subjects are summarized in Table 1. No significant differences were observed in age or ovarian reserve (determined by AMH levels and basal FSH). Approximately one-third of the women had a history of ovarian surgery. Table 2 represents the ovarian stimulation and laboratory outcomes. The duration of stimulation, the total dose of FSH, and the estradiol level on the triggering day were similar. The total FSH dose by the number of oocytes retrieved was comparable between the two groups. However, the total FSH dose by the number of mature oocytes retrieved was significantly lower in the dual group than in the hCG group (634 $\pm 480 \mathrm{IU}$ vs. $982 \pm 748 \mathrm{IU}$, respectively; $p=0.030$ ). Although the number of total oocytes retrieved was statistically similar $(5.3 \pm 3.5$

Table 1. Clinical characteristics of the study subjects

\begin{tabular}{lccc}
\hline Variable & Dual trigger $(\mathrm{n}=40)$ & hCG trigger $(\mathrm{n}=36)$ & $p$-value \\
\hline Age $(\mathrm{yr})$ & $30.9 \pm 4.2$ & $29.6 \pm 4.0$ & 0.174 \\
Body mass index $\left(\mathrm{kg} / \mathrm{m}^{2}\right)$ & $20.0 \pm 2.3$ & $20.4 \pm 2.6$ & 0.361 \\
Basal AMH $(\mathrm{ng} / \mathrm{mL})$ & $0.64 \pm 0.35$ & $0.57 \pm 0.36$ & 0.413 \\
Basal FSH $(\mathrm{IU} / \mathrm{L})$ & $14.6 \pm 16.0$ & $13.9 \pm 14.5$ & 0.863 \\
Basal LH $(\mathrm{mlU} / \mathrm{mL})$ & $8.5 \pm 10.4$ & $5.5 \pm 3.0$ & 0.248 \\
Basal TSH $(\mu \mathrm{lU} / \mathrm{mL})$ & $1.7 \pm 1.0$ & $1.4 \pm 0.6$ & 0.143 \\
Basal prolactin $(\mathrm{ng} / \mathrm{mL})$ & $19.3 \pm 24.5$ & $17.4 \pm 14.4$ & 0.754 \\
Basal estradiol $(\mathrm{pg} / \mathrm{mL})$ & $57.8 \pm 45.9$ & $52.4 \pm 20.3$ & 0.586 \\
Basal AFC & $6.2 \pm 3.3$ & $5.4 \pm 2.6$ & 0.281 \\
Previous ovarian surgery & $35.0(14 / 40)$ & $33.3(12 / 36)$ & 0.878 \\
USG diagnosis of ovarian cyst & $10.0(4 / 40)$ & $22.2(8 / 36)$ & 0.145 \\
\hline
\end{tabular}

Values are presented as mean \pm standard deviation or percent (number). Basal values were measured at day 2 or 3 of the menstrual cycle. hCG, human chorionic gonadotropin; AMH, anti-Müllerian hormone; FSH, follicle-stimulating hormone; LH, luteinizing hormone; TSH, thyroid-stimulating hormone; AFC, antral follicle count; USG, ultrasonography.

Table 2. Stimulation and laboratory results of the study subjects

\begin{tabular}{lccr}
\hline Variable & Dual trigger $(\mathrm{n}=40)$ & hCG trigger $(\mathrm{n}=36)$ & $p$-value \\
\hline Gonadotropin combination & & & 0.628 \\
$\quad$ rFSH only (\%) & $50.0(20 / 40)$ & $55.6(20 / 36)$ & $44.4(16 / 36)$ \\
$\quad$ rFSH+hMG (\%) & $50.0(20 / 40)$ & $7.7 \pm 2.0$ & 0.440 \\
Duration of stimulation (day) & $7.3 \pm 2.1$ & $1,874 \pm 829$ & 0.207 \\
Total dose of FSH used (IU) & $1,658 \pm 642$ & $536 \pm 490$ & 0.439 \\
FSH dose/oocyte & $457 \pm 389$ & $982 \pm 748$ & 0.030 \\
FSH dose/mature oocyte & $634 \pm 480$ & $981 \pm 548$ & 0.875 \\
Estradiol on triggering day (pg/mL) & $1,007 \pm 597$ & $5.0 \pm 2.7$ & 0.655 \\
Number of total oocytes retrieved & $5.3 \pm 3.5$ & $2.3 \pm 1.7$ & 0.010 \\
Number of mature oocytes retrieved & $3.7 \pm 2.7$ & $1.7(3 / 180)$ & 0.336 \\
Degenerative oocyte rate & $0.5(1 / 213)$ & $45.6(82 / 180)$ & $<0.001$ \\
Oocyte maturation rate & $68.5(146 / 213)$ & \\
\hline
\end{tabular}

Values are presented as percent (number) or mean \pm standard deviation.

hCG, human chorionic gonadotropin; rFSH, recombinant follicle-stimulating hormone; hMG, human menopausal gonadotrophin; FSH, follicle-stimulating hormone. 
vs. $5.0 \pm 2.7, p=0.655$ ), the number of mature oocytes retrieved was significantly higher in the dual group than in the $\mathrm{hCG}$ group $(3.7 \pm$ 2.7 vs. $2.3 \pm 1.7$, respectively; $p=0.010$ ). Furthermore, the oocyte maturation rate was significantly higher in the dual group than in the hCG group (68.5\% vs. $45.6 \%$, respectively; $p<0.001)$. OHSS did not occur in either group.

Univariate correlation analysis was used to determine factors significantly related to the number of mature oocytes retrieved: basal $\operatorname{AMH}(p=0.041)$, basal FSH $(p=0.014)$, basal antral follicle count (AFC) $(p<0.001)$, and the dual trigger versus the hCG trigger method $(p=0.011)$. In the multivariate analysis, basal AFC $(p<0.001)$ and the use of the dual trigger method $(p=0.016)$ remained significant factors (Table 3).

\section{Discussion}

The present study showed an increased number of mature oocytes retrieved and higher oocyte maturation rates with the use of a dual trigger in young women with DOR undergoing GnRH antagonist-downregulated $\operatorname{COS}$ cycles. A dual trigger in elective oocyte cryopreservation for young women with DOR is a viable treatment to cryopreserve a large number of mature oocytes in FP. Single women could achieve reproductive autonomy via elective oocyte cryopreservation. Currently, women with DOR consider elective oocyte cryopreservation before compromising their fertility. Young women with DOR have fewer oocytes retrieved, but the possibility of a high-quality embryo and clinical pregnancy are higher once the oocytes are acquired [18]. Although young women with low AMH levels are challenging subjects for oocyte retrieval due to their poor response to COS [19], they should be counseled about efficient oocyte cryopreservation plans. Efficient oocyte cryopreservation could diminish the pressure on young women to have a child or select a partner at a certain time.

In DOR patients, results regarding the number of mature oocytes following dual trigger compared with a trigger of $\mathrm{hCG}$ alone remain inconsistent. Zhang et al. [15] concurred that a dual-trigger approach was beneficial in women with a mean age of 36 years. However, Lin et al. [16] showed no difference in mature oocyte outcomes in women (mean age, 38 years) with DOR undergoing a dual-trigger treatment method. Our study was performed in relatively young women; therefore, a dual-trigger method could be more effective in younger women.

Women undergoing FP want to eventually have a healthy child with their cryopreserved mature oocytes. Although we cannot provide results for embryo quality according to the trigger method, the reproductive outcomes of oocytes treated with a dual-trigger approach do not seem to be compromised. Thorne et al. [20] recently reported that the embryo aneuploidy rate was similar in patients stimulated with a GnRH agonist trigger and patients administered an hCG trigger. Embryo development and quality appear to be unaffected in GnRH agonist trigger cycles [21]. Moreover, a meta-analysis revealed that patients treated with a dual-trigger approach had significantly more good-quality embryos than patients treated with an hCG trigger [22].

The endogenous gonadotropin surge released by the administration of $\mathrm{GnRH}$ agonist, similar to the natural cycle surge, is considered more physiological than the hCG trigger. The importance of a mid-cycle FSH surge induced by a GnRH agonist has been determined in several animal and molecular biology studies. Animal studies have confirmed the importance of FSH in the upregulation of $\mathrm{LH}$ receptor sites formation in granulosa cells $[23,24]$. FSH also plays a crucial role in promoting the resumption of oocyte meiosis $[25,26]$ and the expansion of cumulus cells $[13,27]$. Furthermore, the expression of messenger RNA of reproduction-related genes such as am-

Table 3. Correlation analysis of factors related to the number of mature oocytes retrieved

\begin{tabular}{|c|c|c|c|c|}
\hline \multirow{2}{*}{ Variable } & \multicolumn{2}{|c|}{ Univariate analysis } & \multicolumn{2}{|c|}{ Multivariate analysis } \\
\hline & B & $p$-value & B & $p$-value \\
\hline Female age (yr) & -0.025 & 0.709 & & \\
\hline Body mass index $\left(\mathrm{kg} / \mathrm{m}^{2}\right)$ & -0.203 & 0.075 & & \\
\hline Basal AMH (ng/mL) & 1.607 & 0.041 & 0.071 & 0.937 \\
\hline Basal FSH (IU/L) & -0.050 & 0.014 & -0.030 & 0.263 \\
\hline Basal AFC & 0.396 & $<0.001$ & 0.374 & $<0.001$ \\
\hline Gonadotropin combination, rFSH+ hMG (vs. rFSH) & -0.897 & 0.102 & & \\
\hline Duration of stimulation (day) & 0.064 & 0.64 & & \\
\hline Total dose of FSH used (IU) & 0.001 & 0.412 & & \\
\hline Triggering method, dual trigger (vs. hCG trigger) & 1.372 & 0.011 & 1.36 & 0.016 \\
\hline
\end{tabular}

Basal values were measured on day 2 or 3 of the menstrual cycle.

B, correlation coefficient; $A M H$, anti-Müllerian hormone; FSH, follicle-stimulating hormone; AFC, antral follicle count; $r F S H$, recombinant FSH; hMG, human menopausal gonadotrophin; hCG, human chorionic gonadotropin. 
phiregulin and epiregulin was found to be higher in granulosa cells after a dual-trigger treatment compared to an hCG trigger [28]. Amphiregulin and epiregulin are epidermal growth factor receptor ligands that have been reported to play essential roles in cumulus expansion, oocyte maturation, and meiosis resumption [29,30].

When planning FP, the potential complications and cost of oocyte retrieval should be taken into consideration. In our study, no OHSS or other complications occurred in either group. Although the total dose of FSH used was similar, the efficiency in terms of FSH dose per retrieved mature oocyte was much better with the dual trigger. Further study is needed to evaluate whether the dual-trigger approach is more cost-effective in FP.

We subanalyzed the data according to the gonadotropin combinations (rFSH only or rFSH+hMG; data not shown in the results). The oocyte maturation rates were significantly higher in the dual-trigger group regardless of the COS regimen (rFSH only, $69.5 \%$ vs. $45.8 \%$, $p<0.001$; rFSH with hMG, $67.4 \%$ vs. $45.0 \%, p=0.006$ ). The number of mature oocytes retrieved was higher with the dual-trigger approach, but the difference was not statistically significant due to the small numbers involved (rFSH only: $4.1 \pm 2.6$ vs. $2.8 \pm 1.9, p=0.127$; rFSH with hMG: $3.2 \pm 2.8$ vs. $1.7 \pm 1.4, p=0.089$ ). The dual-trigger approach for final oocyte maturation seemed to improve mature oocyte retrieval regardless of the combination of gonadotropins used.

This study had a few limitations, such as the small number of study subjects and the retrospective nature of the study. Moreover, only the result of mature oocyte cryopreservation was reported; the results after cryopreservation of in vitro matured oocytes were not presented. To the best of our knowledge, this is the first study comparing a dual trigger to an hCG-only trigger in elective oocyte cryopreservation for young women with DOR. The beneficial effect of the dual trigger with a GnRH agonist and hCG on obtaining mature oocytes enabled more mature oocyte cryopreservation in young women with DOR. Future prospective randomized controlled studies are required to validate our findings.

\section{Conflict of interest}

No potential conflict of interest relevant to this article was reported.

\section{ORCID}

Se Jeong Kim
Tae Hyung Kim
Jae Kyun Park
Jin Hee Eum
Woo Sik Lee

https://orcid.org/0000-0003-1879-3451 https://orcid.org/0000-0002-2874-8884 https://orcid.org/0000-0002-5333-4990 https://orcid.org/0000-0002-7875-1870 https://orcid.org/0000-0002-2329-1774
Sang Woo Lyu

https://orcid.org/0000-0001-5572-0898

\section{Author contributions}

Conceptualization: SJK, SWL. Data curation: THK, JKP, JHE. Formal analysis: SJK, THK. Methodology: SJK, WSL, SWL. Project administration: SWL. Visualization: SJK. Writing-original draft: SJK. Writing-review \& editing: SJK, WSL, SWL.

\section{References}

1. Doyle JO, Richter KS, Lim J, Stillman RJ, Graham JR, Tucker MJ. Successful elective and medically indicated oocyte vitrification and warming for autologous in vitro fertilization, with predicted birth probabilities for fertility preservation according to number of cryopreserved oocytes and age at retrieval. Fertil Steril 2016; 105:459-66.

2. Cobo A, Garcia-Velasco JA, Coello A, Domingo J, Pellicer A, Remohi J. Oocyte vitrification as an efficient option for elective fertility preservation. Fertil Steril 2016;105:755-64.

3. Practice Committees of the American Society for Reproductive Medicine and the Society for Assisted Reproductive Technology. Mature oocyte cryopreservation: a guideline. Fertil Steril 2013; 99:37-43.

4. Khalili MA, Shahedi A, Ashourzadeh S, Nottola SA, Macchiarelli G, Palmerini MG. Vitrification of human immature oocytes before and after in vitro maturation: a review. J Assist Reprod Genet 2017;34:1413-26.

5. Practice Committee of the American Society for Reproductive Medicine. Testing and interpreting measures of ovarian reserve: a committee opinion. Fertil Steril 2015;103:e9-17.

6. Jirge PR. Poor ovarian reserve. J Hum Reprod Sci 2016;9:63-9.

7. Orvieto R. Triggering final follicular maturation: hCG, GnRH-agonist or both, when and to whom? J Ovarian Res 2015;8:60.

8. Itskovitz-Eldor J, Kol S, Mannaerts B. Use of a single bolus of GnRH agonist triptorelin to trigger ovulation after $\mathrm{GnRH}$ antagonist ganirelix treatment in women undergoing ovarian stimulation for assisted reproduction, with special reference to the prevention of ovarian hyperstimulation syndrome: preliminary report: short communication. Hum Reprod 2000;15:1965-8.

9. Griesinger G, Diedrich K, Devroey P, Kolibianakis EM. GnRH agonist for triggering final oocyte maturation in the GnRH antagonist ovarian hyperstimulation protocol: a systematic review and meta-analysis. Hum Reprod Update 2006;12:159-68.

10. Shapiro BS, Daneshmand ST, Garner FC, Aguirre M, Thomas S. Gonadotropin-releasing hormone agonist combined with a reduced dose of human chorionic gonadotropin for final oocyte matura- 
tion in fresh autologous cycles of in vitro fertilization. Fertil Steril 2008;90:231-3.

11. Lin MH, Wu FS, Lee RK, Li SH, Lin SY, Hwu YM. Dual trigger with combination of gonadotropin-releasing hormone agonist and human chorionic gonadotropin significantly improves the livebirth rate for normal responders in $\mathrm{GnRH}$-antagonist cycles. Fertil Steril 2013;100:1296-302.

12. Griffin D, Feinn R, Engmann L, Nulsen J, Budinetz T, Benadiva C. Dual trigger with gonadotropin-releasing hormone agonist and standard dose human chorionic gonadotropin to improve oocyte maturity rates. Fertil Steril 2014;102:405-9.

13. Strickland S, Beers WH. Studies on the role of plasminogen activator in ovulation: in vitro response of granulosa cells to gonadotropins, cyclic nucleotides, and prostaglandins. J Biol Chem 1976;251:5694-702.

14. Humaidan P, Kol S, Papanikolaou EG; Copenhagen GnRH Agonist Triggering Workshop Group. GnRH agonist for triggering of final oocyte maturation: time for a change of practice? Hum Reprod Update 2011;17:510-24.

15. Zhang J, Wang Y, Mao X, Chen Q, Hong Q, Cai R, et al. Dual trigger of final oocyte maturation in poor ovarian responders undergoing IVF/ICSI cycles. Reprod Biomed Online 2017;35:701-7.

16. Lin MH, Wu FS, Hwu YM, Lee RK, Li RS, Li SH. Dual trigger with gonadotropin releasing hormone agonist and human chorionic gonadotropin significantly improves live birth rate for women with diminished ovarian reserve. Reprod Biol Endocrinol 2019;17:7.

17. Poseidon Group (Patient-Oriented Strategies Encompassing IndividualizeD Oocyte Number), Alviggi C, Andersen CY, Buehler K, Conforti A, De Placido G, et al. A new more detailed stratification of low responders to ovarian stimulation: from a poor ovarian response to a low prognosis concept. Fertil Steril 2016;105:1452-3.

18. Chang Y, Li J, Li X, Liu H, Liang X. Egg quality and pregnancy outcome in young infertile women with diminished ovarian reserve. Med Sci Monit 2018;24:7279-84.

19. Oh SR, Choe SY, Cho YJ. Clinical application of serum anti-Mullerian hormone in women. Clin Exp Reprod Med 2019;46:50-9.

20. Thorne J, Loza A, Kaye L, Nulsen J, Benadiva C, Grow D, et al. Euploidy rates between cycles triggered with gonadotropin-releasing hormone agonist and human chorionic gonadotropin. Fertil Steril 2019;112:258-65.
21. Acevedo B, Gomez-Palomares JL, Ricciarelli E, Hernandez ER. Triggering ovulation with gonadotropin-releasing hormone agonists does not compromise embryo implantation rates. Fertil Steril 2006;86:1682-7.

22. Chen CH, Tzeng CR, Wang PH, Liu WM, Chang HY, Chen HH, et al. Dual triggering with GnRH agonist plus hCG versus triggering with hCG alone for IVF/ICSI outcome in GnRH antagonist cycles: a systematic review and meta-analysis. Arch Gynecol Obstet 2018; 298:17-26.

23. Richards JS, Ireland JJ, Rao MC, Bernath GA, Midgley AR Jr, Reichert LE Jr. Ovarian follicular development in the rat: hormone receptor regulation by estradiol, follicle stimulating hormone and luteinizing hormone. Endocrinology 1976;99:1562-70.

24. Zeleznik AJ, Midgley AR Jr, Reichert LE Jr. Granulosa cell maturation in the rat: increased binding of human chorionic gonadotropin following treatment with follicle-stimulating hormone in vivo. Endocrinology 1974;95:818-25.

25. Yding Andersen C, Leonardsen L, Ulloa-Aguirre A, Barrios-De-Tomasi J, Moore L, Byskov AG. FSH-induced resumption of meiosis in mouse oocytes: effect of different isoforms. Mol Hum Reprod 1999;5:726-31.

26. Zelinski-Wooten MB, Hutchison JS, Hess DL, Wolf DP, Stouffer RL. Follicle stimulating hormone alone supports follicle growth and oocyte development in gonadotrophin-releasing hormone antagonist-treated monkeys. Hum Reprod 1995;10:1658-66.

27. Eppig JJ. FSH stimulates hyaluronic acid synthesis by oocyte-cumulus cell complexes from mouse preovulatory follicles. Nature 1979;281:483-4.

28. Haas J, Ophir L, Barzilay E, Machtinger R, Yung Y, Orvieto R, et al. Standard human chorionic gonadotropin versus double trigger for final oocyte maturation results in different granulosa cells gene expressions: a pilot study. Fertil Steril 2016;106:653-9.

29. Caixeta ES, Machado MF, Ripamonte P, Price C, Buratini J. Effects of FSH on the expression of receptors for oocyte-secreted factors and members of the EGF-like family during in vitro maturation in cattle. Reprod Fertil Dev 2013;25:890-9.

30. Park JY, Su YQ, Ariga M, Law E, Jin SL, Conti M. EGF-like growth factors as mediators of LH action in the ovulatory follicle. Science 2004;303:682-4. 